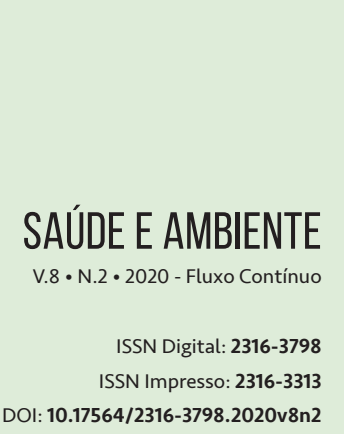

DOI: $10.17564 / 2316-3798.2020 v 8 n 2$

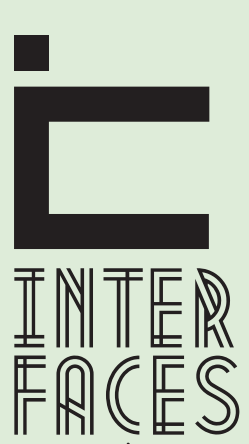

CIENTÍFICAS

\section{SÍNDROME DE BURNOUT E FATORES DE ESTRESSE LABORAL EM PROFESSORES DE ESCOLAS PÚBLICAS DO MUNICÍPIO DE CAPÃO DO LEÃO, RS}

BURNOUT SYNDROME AND WORK STRESS FACTORS AMONG PUBLIC SCHOOL TEACHERS IN THE CITY OF CAPÃO DO LEÃO, RS

SÍNDROME DE BURNOUT Y FACTORES DE ESTRÉS LABORAL EN MAESTROS DE ESCUELAS PÚBLICAS DE CAPÃO DO LEÃO, RS

Ingrid Masseron Cunha ${ }^{1}$ Igor Soares Vieira ${ }^{2}$

Matheus dos Santos Fernandez ${ }^{3}$ Ana Laura Sica Cruzeiro Szortyka ${ }^{4}$

\section{RESUMO}

Este estudo transversal avaliou a ocorrência e fatores associados à Síndrome de Burnout (SB) em professores da rede pública do município de Capão do Leão, Rio Grande do Sul, Brasil. Professores de todas as escolas municipais de ensino fundamental da zona urbana do município foram convidados a responder um questionário semiestruturado $(\mathrm{N}=$ 168). 0 instrumento de avaliação foi organizado em blocos, de acordo com as respectivas temáticas, sendo elas: características sociodemográficas; variáveis laborais e Escala Maslach Burnout Inventory $(\mathrm{MBI})$. As diferenças entre as médias dos domínios do $\mathrm{MBI}$ e as variáveis independentes foram testadas pelo Teste t de Student. Um total de 102 professores participaram do estudo. A dimensão "exaustão" foi associada ao sentimento de sobrecarga no trabalho $(p<0,001)$, sobrecarga com a carga horária trabalhada $(p<0,001)$ e insatisfação com o salário $(p=0,006)$. A dimensão "despersonalização" foi associada apenas ao sentimento de sobrecarga com a carga horária trabalhada $(p=0,021)$, enquanto na dimensão "realização pessoal", o único fator associado foi a identificação com a profissão que desempenha atualmente $(p=0,026)$. Este estudo enfatiza a necessidade da criação de políticas e serviços de apoio centrados nos professores de escolas públicas, a fim de promover um contexto mais favorável ao exercício da profissão docente.

\section{PALAVRAS-CHAVE}

Saúde Ocupacional. Professores. Estresse Ocupacional. 


\section{ABSTRACT}

This cross-sectional study evaluated the occurrence and factors associated with Burnout Syndrome (SB) among public school teachers in the city of Capão do Leão, Rio Grande do Sul, Brazil. Teachers from all municipal elementary schools in the urban area of the municipality were invited to answer a semi-structured questionnaire $(\mathrm{N}=168)$. The assessment instrument was organized in blocks, according to the respective themes: sociodemographic characteristics; work variables and Maslach Burnout Inventory Scale (MBI). The differences between the means of the MBI domains and the independent variables were tested by the Student's t test. A total of 102 teachers participated in the study. The "exhaustion" dimension was associated with the feeling of overload at work $(p<0.001)$, overload with the hours worked $(p<0.001)$ and dissatisfaction with the salary $(p=0.006)$. The "depersonalization" dimension was associated with the feeling of being overloaded with the number of hours worked $(p=0.021)$, and the "personal fulfillment" dimension was associated with the identification with the profession $(p=0.026)$. This study emphasizes the need to create support policies and services focused on public school teachers, in order to promote a more favorable context for the exercise of the teaching profession.

\section{KEYWORDS}

Occupational Health, Teachers, Occupational stress.

\section{RESUMEN}

Este estudio transversal evaluó la ocurrencia y los factores asociados al Síndrome de Burnout (SB) en profesores de escuelas públicas de la ciudad de Capão do Leão, Rio Grande do Sul, Brasil. Se invitó a docentes de todas las escuelas primarias municipales del área urbana del municipio a responder un cuestionario semiestructurado $(\mathrm{N}=168)$. El instrumento de evaluación se organizó en bloques, según los temas respectivos, que son: características sociodemográficas; variables laborales y Maslach Burnout Inventory (MBI). Las diferencias entre las medias de los dominios del MBI y las variables independientes se probaron mediante la prueba t de Student. Participaron del estudio un total de 102 profesores. La dimensión “agotamiento” se asoció con la sensación de sobrecarga en el trabajo $(p<0.001)$, sobrecarga con las horas trabajadas $(p<0.001)$ e insatisfacción con el salario $(p=0.006)$. La dimensión "despersonalización" se asoció únicamente al sentimiento de sobrecarga con el número de horas trabajadas ( $p=0.021$ ), mientras que en la dimensión "realización personal”, el único factor asociado fue la identificación con la profesión que actualmente desempeña $(p=0.026)$. Este estudio enfatiza la necesidad de crear políticas y servicios de apoyo enfocados a los docentes de las escuelas públicas, con el fin de promover un contexto más favorable para el ejercicio de la profesión docente. 


\section{PALABRAS CLAVE}

Salud Ocupacional, Maestros, Estrés ocupacional.

\section{INTRODUCÇÃO}

Durante o exercício da profissão docente, inúmeros estressores psicossociais estão presentes, alguns relacionados com a natureza de suas funções, outros associados ao contexto institucional e social (ARONSSON et al., 2017; LUZ et al., 2019). Quando persistentes, esses estressores podem desencadear a Síndrome de Burnout (SB) (CARLOTTO; PALAZZO, 2006; MASLACH; JACKSON, 1981). De acordo com a Organização Mundial da Saúde (OMS), a SB é um problema de saúde pública que se caracteriza como um estado de exaustão vital, sendo um potencial fator de risco para o desenvolvimento de transtornos atrelados à saúde física e mental (OMS, 2000).

A SB é definida como uma síndrome psicológica, envolvendo três dimensões: exaustão emocional, despersonalização e uma diminuição na sensação de realização pessoal (MASLACH; JACKSON, 1981; MASLACH et al., 1986; TETRICK et al., 2005). A exaustão emocional é a falta de energia e entusiasmo, por sensação de esgotamento de recursos ao qual pode somar-se o sentimento de frustração e tensão nos trabalhadores, enquanto a despersonalização é caracterizada pelo desenvolvimento de uma insensibilidade emocional, que faz com que o profissional trate os indivíduos, colegas e a organização de maneira desumanizada.

A diminuição da realização pessoal no trabalho pode ser compreendida como uma tendência do trabalhador a auto avaliar-se de forma negativa, tornando-se infeliz e insatisfeito com seu desenvolvimento profissional, com consequente declínio no seu sentimento de competência e êxito (MASLACH et al., 1986).

A severidade da SB entre os profissionais de ensino é superior àquela encontrada entre os profissionais de saúde, o que destaca o magistério como uma das profissões de alto risco para o desenvolvimento da SB (CARLOTTO, 2011). Determinados fatores são capazes de gerar estresse crônico entre professores escolares, como a sobrecarga de trabalho, desempenho de tarefas administrativas complementares, salas de aula superlotadas, problemas de disciplina de classe, conflitos com superiores, colegas de trabalho e familiares, reformas educacionais contínuas, déficits na formação, promoção e desenvolvimento profissional, baixos salários, além de problemas institucionais de infraestrutura e organização (DUKE et al., 2020; LUZ et al., 2019).

Todavia, esses desafios vivenciados pelos professores são variáveis de acordo com as características sociodemográficas, condições estruturais e organizacionais do contexto de trabalho em que esses indivíduos estão inseridos (BATISTA et al., 2010; CARLOTTO; PALAZZO, 2006; MESSIAS et al., 2019). Neste sentido, este estudo investigou a ocorrência de SB em professores de escolas públicas de ensino fundamental no município de Capão do Leão, Rio Grande do Sul, Brasil, bem como verificou associações com variáveis laborais e fatores de estresse percebidos no trabalho. 


\section{MÉTODO}

\subsection{DESENHO DO ESTUDO}

A população-alvo deste estudo transversal foi composta por professores do ensino fundamental de escolas públicas urbanas do município de Capão do Leão, Rio Grande do Sul, Brasil. A coleta de dados ocorreu no período entre março e agosto de 2019.

\subsection{AMOSTRA}

Professores de todas as sete escolas da rede municipal de ensino fundamental da zona urbana do município de Capão do Leão foram convidados a participar da pesquisa. Foram incluídos na amostra professores que atuavam há mais de 5 anos no magistério e que concordaram em participar do estudo. Ademais, foram excluídos da amostra aqueles professores escolares que estavam ausentes das atividades de trabalho em virtude de problemas de saúde.

\subsection{INSTRUMENTO DE PESQUISA}

O questionário de pesquisa deste estudo foi organizado em diferentes blocos, sendo eles: (I) Variáveis independentes: características sociodemográficas; práticas de atividade física; percepção de autovalorização no ambiente de trabalho; estressores laborais; (II) Desfecho: Escala Maslach Burnout Inventory.

\subsection{VARIÁVEIS INDEPENDENTES}

As características sociodemográficas compreenderam as seguintes variáveis: "faixa-etária" ( $\leq 38$ anos; 39 a 46 anos; 47-52 anos; $\geq 53$ anos) e "estado civil" (solteiro(a); casado / vive com o(a) companheiro(a); separado(a)/divorciado(a); viúvo(a). A classificação socioeconômica foi mensurada de acordo com os critérios estabelecidos pela classificação da Associação Brasileira de Empresas de Pesquisa (ABEP), que propõe um sistema de pontos (escores) para aferir o poder de compra das famílias urbanas categorizadas em cinco classes (BRASIL, 2016). Para fins de análise estatística, os escores foram agrupados da seguinte forma, de acordo com a variação (mínimo - máximo) da renda familiar média: $A+B$ (R\$2.013,00 - R\$ 9.733,00); C (R\$ 726 - R\$ 1.195,00); D+E (R\$ 277,00 - R\$ 485,00). A variável que investigou se os professores possuem filhos considerou indivíduos que eram pais e não pais (sim; não).

A avaliação da prática de atividades físicas entre os professores identificou indivíduos sedentários (não praticam nenhum exercício físico durante a semana) e ativos (prática rotineira de exercício físico durante a semana). O sentimento de autovalorização no ambiente de trabalho foi verificado por meio de uma questão objetiva com opção dicotômica de resposta (sim; não). Os estressores laborais avaliados pelo estudo são apresentados no Quadro 1. Uma série de sentenças afirmativas foram apresentadas aos entrevistados, sendo que estes deveriam destacar a opção de resposta condizente com a sua realidade no ambiente de trabalho (sim; não). 
Quadro 1 - Variáveis referentes aos estresses laborais. Capão do Leão, Brasil, 2020

\begin{tabular}{|} 
Sentimento de sobrecarrega no trabalho \\
Sentimento de sobrecarrega com a carga horária trabalhada \\
Sentimento de exigência no que se refere ao alto desempenho na sala de aula \\
Identificação com a profissão a qual desempenha atualmente \\
Satisfação com o salário \\
Sentimento que desenvolve mais tarefas que deveria \\
Ter aluno com deficiência em sala de aula \\
Alunos indisciplinados em sala de aula \\
Sentir-se exigido \\
Sentir-se apoiado pelos colegas \\
Sentir-se apoiado pela família \\
Sentir-se motivado em ir para a escola
\end{tabular}

Fonte: Dados da pesquisa.

\subsection{DESFECHO}

Para verificar a ocorrência da SB, foi utilizada a versão brasileira da Escala Maslach Burnout Inventory (MBI) para educadores (CAMPOS; MAROCO, 2012). Esta versão do instrumento é composta por 22 questões, cuja opção de resposta pode variar de 1 a 5, onde: 1: nunca; 2: algumas vezes ao ano; 3: algumas vezes no mês; 4: algumas vezes na semana; 5: diariamente. Os domínios avaliados pelo MBI são: “exaustão emocional” (itens 1-3, 6-8, 13, 14, 16, 20), “despersonalização" (itens 5, 10, 11, $15,22)$ e "realização pessoal" (itens $4,7,9,12,17,18,19,21)$. Altos escores em "exaustão emocional" e "despersonalização" correspondem a um elevado nível de SB, enquanto baixos escores em "realização profissional” também estão relacionados a alto nível da SB.

\subsection{ANÁLISE ESTATÍSTICA}

Os dados foram duplamente digitados e validados no EpiData (versão 3.1). A análise estatística foi realizada por meio do software Statistical Package for the Social Sciences (IBM ${ }^{\circledR}$ SPSS 25.0; Chicago, USA). 0 teste de Shapiro-Wilk foi adotado para verificar a normalidade e demonstrou que a amostra apresentou distribuição paramétrica (normal). A análise descritiva das variáveis foi observada a partir da distribuição das frequências absolutas e relativas, tendência central (média) e variabilidade (desvio-padrão). As diferenças entre as médias das dimensões do MBI e as variáveis independentes foram testadas pelo Teste $t$ de Student, considerando associações significativas quando $\mathrm{p}$-valor $\leq 0,05$. 


\subsection{ASPECTOSÉTICOS}

Este recebeu parecer favorável do Comitê de Ética em Pesquisa da Faculdade de Medicina da Universidade Federal de Pelotas (\#3111531). Todos participantes que concordaram em participar da pesquisa assinaram o Termo de Consentimento Livre e Esclarecido.

\section{RESULTADOS}

A população-alvo do estudo foi composta por 168 professores escolares, sendo que após a aplicação dos critérios de inclusão, 66 sujeitos foram considerados como perdas ou recusas. Assim, um total de 102 professores foram incluídos na amostra final do estudo, correspondendo a uma taxa de participação de $60,8 \%$.

A análise descritiva apresentada na Tabela 1 evidencia uma distribuição homogênea entre as faixas etárias da amostra. A maioria dos professores era casada, estava incluída nas classes econômicas A e B e possuía filhos.

Tabela 1 - Características sociodemográficas da amostra (n=102). Capão do Leão, Brasil, 2020

\begin{tabular}{ccc}
\hline Variáveis & $\mathbf{n}$ & \% \\
\hline Idade & & \\
$\leq 38$ anos & 23 & 22,5 \\
39 a 46 anos & 26 & 25,5 \\
47 a 52 anos & 26 & 25,5 \\
$\geq 53$ & 27 & 26,5 \\
Estado civil & & \\
Solteiro(a) & 17 & 16,7 \\
Casado(a) / vive com companheiro(a) & 69 & 67,6 \\
Separado(a) / divorciado(a) & 13 & 12,7 \\
Viúvo (a) & 3 & 2,9 \\
Classe socioeconômica & & \\
A e B & 58 & 56,9 \\
C & 35 & 34,3 \\
De E & 9 & 8,8 \\
\hline
\end{tabular}




\section{Possuir filhos}

Não

Sim
26

76
25,5

74,5

Fonte: Dados da pesquisa

Os professores apresentaram as maiores médias nas dimensões “realização pessoal” e "exaustão emocional" (34,57 $\pm 8,32$ e $29,13 \pm 12,12$, respectivamente). A pontuação mais baixa foi verificada na dimensão "despersonalização" $(6,97 \pm 6,18)$ (FIGURA 1).

As dimensões do MBI "realização pessoal" e "exaustão emocional" apresentaram as maiores médias (34,57 $\pm 8,32$ e $29,13 \pm 12,12$, respectivamente), conforme apresentado na Figura 1. A pontuação média mais baixa foi verificada na dimensão "despersonalização" $(6,97 \pm 6,18)$.

Figura 1 - Índices médios das dimensões avaliadas pela escala Maslach Burnout Inventory $(\mathrm{n}=102)$. Capão do Leão, Brasil, 2020

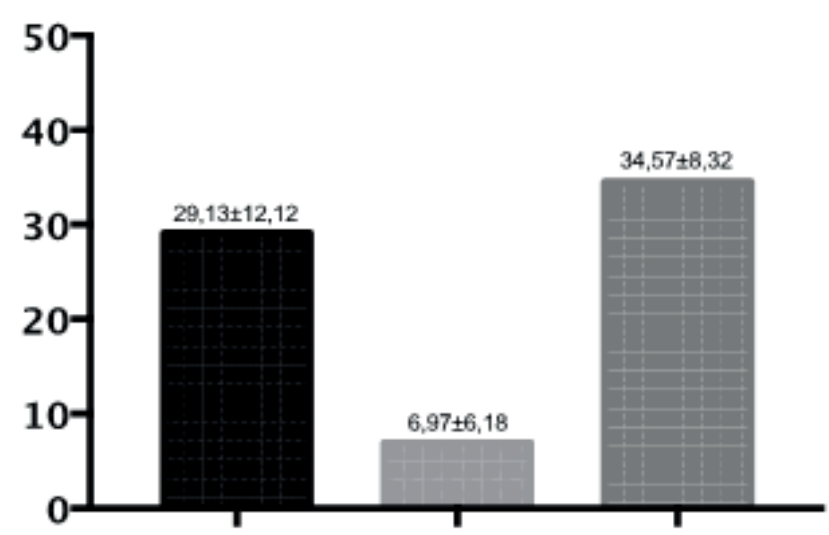

\section{$\square$ Cansaço emocional Despersonalização Realização pessoal}

Fonte: Dados da pesquisa.

Quando verificada a relação entre as dimensões da SB com as variáveis da Tabela 2, foi encontrada as seguintes associações significativas: a maior média na dimensão "exaustão emocional" foi observada entre os indivíduos sedentários $(p=0,030)$ e que não possuem sentimentos autovalorização $(p<0,001)$. Além disso, a dimensão "realização pessoal" esteve associada apenas com o sentimento de autovalorização $(p=0,005)$. 
Tabela 2 - Dimensões da escala MBI de acordo com ter filhos, atividade física e autovalorização ( $\mathrm{n}=$ 102). Capão do Leão, Brasil, 2020

\begin{tabular}{ccccccc}
\hline Variáveis & $\begin{array}{c}\text { Exaustão } \\
\text { emocional } \\
\text { Média } \pm \text { DV }\end{array}$ & p* & $\begin{array}{c}\text { Despersonalização } \\
\text { Média } \pm \text { DV }\end{array}$ & p* & $\begin{array}{c}\text { Realização } \\
\text { pessoal } \\
\text { Média } \pm \text { DV }\end{array}$ & $\mathbf{p}^{*}$ \\
\hline Possuir filhos & & & & & & 0,794 \\
Não & $29,57 \pm 11,96$ & 0,826 & $7,73 \pm 7,33$ & 0,523 & $34,92 \pm 7,78$ & \\
Sim & $28,97 \pm 12,24$ & & $6,71 \pm 5,76$ & & $34,44 \pm 8,53$ & \\
Atividade Física & & & & & & \\
Sedentário & $31,60 \pm 12,59$ & 0,030 & $7,09 \pm 6,31$ & 0,834 & $33,28 \pm 9,05$ & 0,105 \\
Ativo & $26,44 \pm 11,09$ & & $6,83 \pm 6,09$ & & $35,95 \pm 8,45$ & \\
Autovalorização & & & & & & \\
Não & $33,08 \pm 11,23$ & $<0,001$ & $7,61 \pm 6,28$ & 0,316 & $32,18 \pm 8,46$ & 0,005 \\
Sim & $25,47 \pm 11,85$ & & $6,37 \pm 6,08$ & & $37,77 \pm 7,60$ & \\
\hline
\end{tabular}

Legenda: MBI: Maslach Burnout Inventory; DV: desvio padrão; *Teste t de Student.

Fonte: Dados da pesquisa.

Com relação aos fatores de estresse percebidos pelos professores, verificou-se que a média de "exaustão emocional" foi maior entre os professores que tiveram sentimentos de sobrecarga no trabaIho $(p<0,001)$, sobrecarga com carga horária trabalhada $(p<0,001)$ e entre àqueles que não estavam satisfeitos com o salário $(p=0,006)$. A identificação com a profissão foi associada a maior média na dimensão “realização pessoal” $(p=0,026)$. Na dimensão “despersonalização”, a sobrecarga com a carga horária trabalhada foi associada a esta dimensão ( $p=0,021)$ (TABELA 3).

Tabela 3 - Dimensões da escala MBI de acordo com os sentimentos relacionados ao trabalho e carga horária ( $n=102)$. Capão do Leão, Brasil, 2020

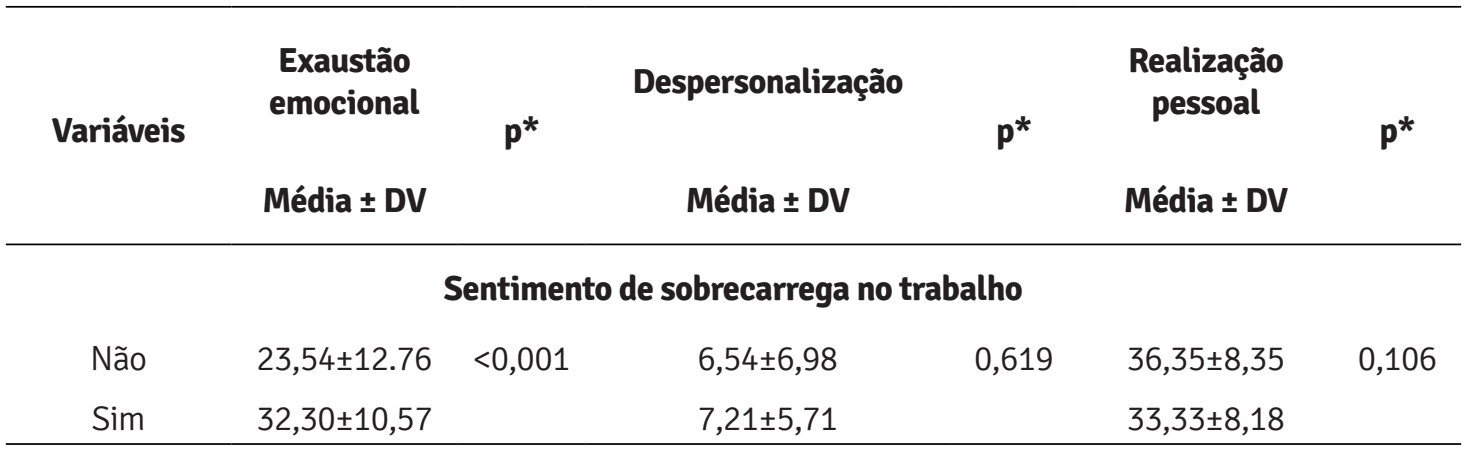




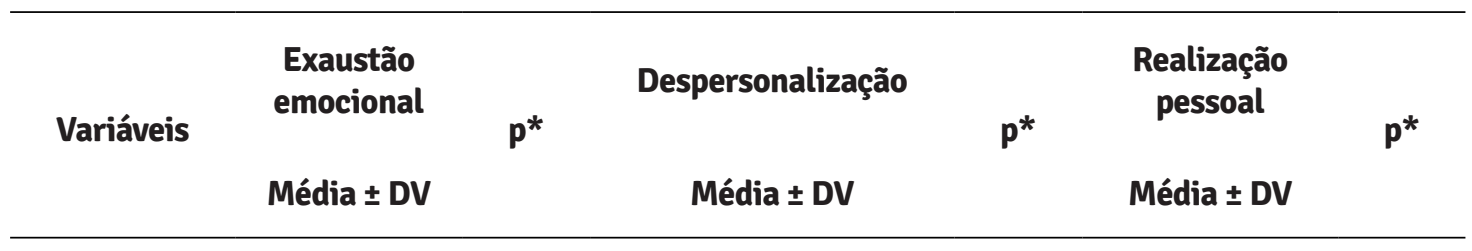

Sentimento de sobrecarrega com a carga horária trabalhada

$\begin{array}{lllllll}\text { Não } & 23,58 \pm 13,11 & <0,001 & 5,23 \pm 5,63 & 0,021 & 37,46 \pm 8,01 & 0,005 \\ \text { Sim } & 32,55 \pm 10,13 & & 8,04 \pm 6,30 & & 32,77 \pm 8,04 & \end{array}$

Sentimento de exigência no que se refere ao alto desempenho na sala de aula

$\begin{array}{lllllll}\text { Não } & 28,88 \pm 13,65 & 0,886 & 6,55 \pm 7,72 & 0,677 & 36,50 \pm 9,43 & 0,097 \\ \text { Sim } & 29,25 \pm 11,37 & & 7,17 \pm 5,29 & & 33,60 \pm 7,59 & \end{array}$

\section{Identificação com a profissão a qual desempenha atualmente}

\begin{tabular}{ccccccc} 
Não & $31,9 \pm 12,72$ & 0,265 & $7,42 \pm 6,14$ & 0,705 & $30,19 \pm 9,99$ & 0,026 \\
Sim & $28,40 \pm 11,93$ & \multicolumn{5}{c}{$3,85 \pm 6,22$} \\
& & \multicolumn{5}{c}{ Satisfação com o salário } \\
Não & $31,47 \pm 11,89$ & 0,006 & $7,32 \pm 6,08$ & 0,386 & $34,62 \pm 8,28$ & 0,937 \\
Sim & $24,45 \pm 11,17$ & & $6,16 \pm 6,52$ & & $34,48 \pm 8,66$ &
\end{tabular}

\section{Sentimento que desenvolve mais tarefas que deveria}

\begin{tabular}{lllllll} 
Não & $26,17 \pm 13,20$ & 0,057 & $6,35 \pm 7,01$ & 0,473 & $36,28 \pm 7,79$ & 0,120 \\
Sim & $31,11 \pm 11,12$ & & $7,32 \pm 5,77$ & & $33,60 \pm 8,44$ & \\
\hline
\end{tabular}

Legenda: MBI: Maslach Burnout Inventory; DV: desvio padrão; *Teste t de Student.

Fonte: Dados da pesquisa

As dimensões avaliadas pelo MBI de acordo com os sentimentos relacionados aos alunos, colegas de trabalho, família e motivação de ir à escola, são apresentados na Tabela 4. A dimensão "exaustão emocional" apresentou associação com a indisciplina dos alunos $(p=0,002)$, sentir-se exigido $(p=0,049)$ e falta de motivação em ir à escola $(p<0,001)$. Na dimensão “despersonalização", estiveram associados os seguintes fatores de estresse: indisciplina dos alunos $(p<0,001)$, sentir-se exigido $(p=0,004)$ e falta de motivação em ir à escola $(p<0,001)$. A “realização pessoal” também foi associada a ter alunos indisciplinados em sala de aula e a falta de motivação em ir à escola $(p=0,003)$. 
Tabela 4 - Dimensões da escala MBI de acordo com os sentimentos relacionados aos alunos, colegas de trabalho, família e motivação de ir à escola (n=102). Capão do Leão, Brasil, 2020

\begin{tabular}{|c|c|c|c|c|c|c|}
\hline Variáveis & $\begin{array}{c}\text { Exaustão } \\
\text { emocional } \\
\text { Média } \pm \text { DV }\end{array}$ & $\mathbf{p}^{*}$ & $\begin{array}{c}\text { Despersonalização } \\
\text { Média — DV }\end{array}$ & p* & $\begin{array}{c}\text { Realização } \\
\text { pessoal } \\
\text { Média } \pm \text { DV }\end{array}$ & p* \\
\hline \multicolumn{7}{|c|}{ Ter aluno com deficiência em sala de aula } \\
\hline Não & $26,40 \pm 12,00$ & 0,099 & $8,73 \pm 7,18$ & 0,293 & $32,26 \pm 7,26$ & 0,211 \\
\hline Sim & $30,44 \pm 12,09$ & & $6,60 \pm 5,83$ & & $34,96 \pm 8,29$ & \\
\hline \multicolumn{7}{|c|}{ Alunos indisciplinados em sala de aula } \\
\hline Não & $23,87 \pm 11,75$ & 0,002 & $4,42 \pm 4,45$ & $<0,001$ & $38,45 \pm 6,90$ & $<0,001$ \\
\hline Sim & $31,63 \pm 11,54$ & & $8,18 \pm 6,53$ & & $32,71 \pm 8,33$ & \\
\hline \multicolumn{7}{|c|}{ Sentir-se exigido } \\
\hline Não & $24,22 \pm 13,53$ & 0,049 & $4,27 \pm 4,18$ & 0,004 & $37,09 \pm 6,84$ & 0,095 \\
\hline Sim & $30,69 \pm 11,32$ & & $7,73 \pm 6,48$ & & $33,74 \pm 8,56$ & \\
\hline \multicolumn{7}{|c|}{ Sentir-se apoiado pelos colegas } \\
\hline Não & $37,12 \pm 11,45$ & 0,051 & $12,00 \pm 7,75$ & 0,160 & $32,37 \pm 7,04$ & 0,440 \\
\hline Sim & $28,44 \pm 11,98$ & & $6,54 \pm 6,05$ & & $34,75 \pm 8,42$ & \\
\hline \multicolumn{7}{|c|}{ Sentir-se apoiado pela família } \\
\hline Não & $24,83 \pm 9,45$ & 0,175 & $8,33 \pm 7,68$ & 0,668 & $34,00 \pm 13,16$ & 0,864 \\
\hline Sim & $29,33 \pm 12,41$ & & $6,88 \pm 6,11$ & & $34,60 \pm 8,02$ & \\
\hline \multicolumn{7}{|c|}{ Sentir-se motivado em ir para a escola } \\
\hline Não & $40,08 \pm 10,31$ & $<0,001$ & $10,88 \pm 7,35$ & 0,003 & $30,28 \pm 8,61$ & 0,003 \\
\hline Sim & $25,73 \pm 10,42$ & & $5,69 \pm 5,23$ & & $30,85 \pm 7,77$ & \\
\hline
\end{tabular}

Legenda: MBI: Maslach Burnout Inventory; DV: desvio padrão; *Teste t de Student.

Fonte: Dados da pesquisa.

\section{DISCUSSÃO}

Os achados encontrados neste estudo salientam a natureza de caráter exigente e estressante da profissão docente, sendo de extrema importância a verificação do conjunto de tensões exercidas sobre estes profissionais, uma vez que se apresentam bastante variados e distintos (ARONSSON et al., 2017; LEITE et al., 2019; LEVY et al., 2009; LUZ et al., 2019). 
A SB é uma resposta ao estresse laboral crônico com experiência subjetiva de caráter negativo, sendo composta por cognições, emoções e atitudes no ambiente de trabalho (ARONSSON et al., 2017; CARLOTTO, 2011; MASLACH et al., 1986). Segundo o modelo teórico de Maslach e Jackson (1981), a SB caracteriza-se por um processo em que a exaustão emocional é a dimensão precursora da síndrome, seguida pela despersonalização e, por fim, pelo sentimento de diminuição da realização pessoal no trabalho. Nesse contexto, os resultados obtidos no presente estudo apontam que este processo pode estar em desenvolvimento entre os professores avaliados.

Foram encontradas associações significativas que relacionam a exaustão emocional com o sedentarismo. Esse resultado pode ser explicado pelo fato de que a prática de atividades físicas regula 0 impacto do estresse sob a rotina dos indivíduos, visto que o exercício físico não traz apenas benefícios para a saúde física, podendo atuar igualmente na melhoria do bem-estar psicossocial do indivíduo (DREHER et al., 2020). Mandolesi e colaboradores (2018) demonstraram sistematicamente a concordância referente aos benefícios físicos, fisiológicos e psicológicos da prática de atividade física de forma regular e contínua e consideraram que a prática ativa de exercícios físicos pode atuar como fator modificável para prevenção, melhora das habilidades cognitivas e melhora do humor.

Em relação ao sentimento de autovalorização, àqueles professores que não se autovalorizam apresentaram significativamente maior exaustão emocional e diminuição da sua realização pessoal no trabalho. Com isso, é importante destacar que o sentimento de importância e a autovalorização são recursos essenciais aos trabalhadores, pois são necessidades humanas fundamentais para a satisfação no ambiente de trabalho (ARONSSON et al., 2017; ROBBINS et al., 2010). Nesse sentido, sugere-se realizar um trabalho com os professores direcionado para a construção e valorização da sua autoestima, autoconfiança e autoconhecimento, propiciando assim, um ambiente educativo baseado na satisfação, na solidariedade e na generosidade (ARANTES, 2003).

Paralelamente a essa autovalorização do docente, a identificação com a profissão também exerce papel muito importante no bem-estar do professor e no ambiente de trabalho (RAUSCH; DUBIELLA, 2013). O sentimento de insatisfação profissional não afeta somente os próprios professores, mas também o clima e as interações sociais vivenciadas em sala de aula (OUELLETTE et al., 2018).

Nossos resultados identificaram que os professores que não se sentiam motivados em ir à escola apresentaram maiores médias em todas as dimensões do MBI, corroborando estudos prévios (CARLOTTO; CÂMARA, 2004). Gursel e colaboradores (2002) definem satisfação profissional como um estado emocional positivo resultante da situação profissional do sujeito e associado às características e tarefas específicas da profissão.

Esta definição está de acordo com a base teórica de Hackman e Oldham (1975), onde dizem que as condições de trabalho dão origem à motivação e para eles, as tarefas enriquecidas associam-se ao aumento dos níveis de satisfação, produtividade e motivação no trabalho. Assim, vale ressaltar os aspectos centrais sobre o significado do trabalho, onde a responsabilidade por resultados e o feedback recebido em relação ao desempenho são importantes.

A sobrecarga com o trabalho, sobrecarga com a carga horária e sentir-se exigido também foram fatores associados às maiores médias entre as dimensões "exaustão emocional" e "despersonalização” (GURSEL et al., 2002; HACKMAN; OLDHAM, 1975). Durante o exercício da profissão docente, 
além das atividades desenvolvidas na escola, muitos professores levam tarefas para casa, o que acaba comprometendo o tempo disponível para atividades com a família ou para descanso (CHOPRA, 2009).

Um estudo realizado em escolas municipais de João Pessoa (Paraíba, Brasil), corroborando com nossos achados, também revelou que o fato de o professor acreditar que a atividade laboral extrapola os seus limites está relacionado à exaustão emocional (BATISTA et al., 2010). É possível que o excesso de trabalho e a sensação de que este excede o seu lugar, interferindo na vida pessoal, além dos cuidados com a família, provoca sensação de insatisfação e desinteresse pelo trabalho, fazendo com que o professor se perceba inferior e distante da realização profissional. No entanto, vale destacar que as atividades de lazer podem diminuir a vulnerabilidade a SB, visto que pode ser capaz de proporcionar sensação de bem-estar (CARLOTTO, 2002).

A insatisfação salarial também parece ter um impacto importante sobre o estresse e subsequente maior grau de exaustão emocional em nossa amostra. Para Gatti (1996), a ausência de uma remuneração coerente leva os profissionais da educação a acumularem cargos, aumentando de maneira demasiada sua jornada de trabalho. Esse aumento acarreta sérios problemas na qualidade das aulas ministradas, visto que no turno em que o professor poderia estar preparando suas aulas, ele se encontra lecionando em outras escolas para complementar seu orçamento (GATTI, 1996; MENDES, 2015).

Esteves-Ferreira e colaboradores (2014) destacam que, principalmente entre os professores do ensino público, a questão salarial e a jornada de trabalho excessiva se destacaram como os principais desencadeadores de insatisfação. Esses dados também corroboram os resultados de Levy e colaboradores (2009), que mostram a questão salarial como central, por acarretar a necessidade do aumento da jornada de trabalho. Os baixos rendimentos dos professores vêm colaborar duplamente para o adoecimento docente, provocando uma exigência de excesso de trabalho e produzindo o desrespeito da sociedade por esse profissional, para quem onde o valor do dinheiro é quem dita o status da profissão (CARLOTTO, 2011; DUKE et al., 2020; ESTEVES-FERREIRA et al., 2014).

A indisciplina discente é um conjunto de determinadas contrariedades no cotidiano, resultantes de rupturas efetuadas por alunos e que pode ser manifestada por meio de ofensas, atitudes grosseiras, desordem, violência física e, de modo geral, desrespeito (LEWIS, 1999; SMETACKOVA et al., 2019). Em nosso estudo, os professores que referiram ter alunos indisciplinados em sala de aula apresentaram maiores níveis de exaustão emocional, despersonalização e diminuição da realização pessoal. 0 desrespeito sofrido pelo professor influencia negativamente em seu comportamento, sendo fonte de estresse, gerando efeitos diretos na sua saúde física e mental, tornando seu ambiente de trabalho mais difícil e insuportável (CARLOTTO, 2011; SMETACKOVA et al., 2019).

Os alunos representam a maior parte do tempo do trabalho docente, no entanto, se esse relacionamento não for satisfatório, pode tornar-se fonte de grande consumo de energia de um profissional que já está esgotado, além de levar à diminuição de sua eficiência e ao sentimento de insatisfação com o trabalho (CARLOTTO; PALAZZO, 2006; LEVY et al., 2009; SMETACKOVA et al., 2019). Estudos vêm sendo realizados sistematicamente, demonstrando que as dificuldades de relacionamento com os alunos e a indisciplina representem o maior problema que os professores enfrentam no exercício da sua atividade (GOMES et al., 2010). 


\section{CONCLUSÃO}

A constante preocupação com a saúde mental dos professores torna-se importante e evidente, uma vez que os resultados deste estudo evidenciam o estabelecimento da SB nos professores avaliados. Reconhecer essa realidade e promover medidas públicas para assegurar condições de trabalho adequadas, pode melhorar a qualidade de vida desses profissionais e colaborar com a satisfação e autoestima no ambiente de trabalho.

Este estudo enfatiza a necessidade de um contexto mais favorável ao exercício da profissão docente, sugerindo o desenvolvimento de programas que propiciem um espaço institucional de discussão e reflexão, com vistas a minimizar o impacto dos fatores de estresse laboral e melhorar o sentimento de realização profissional entre os professores de ensino fundamental da rede pública de ensino.

Na presença de fatores pessoais predisponentes, condições de trabalho desfavoráveis e relações sociais prejudicadas podem ser consideradas obstáculos ao bom desempenho profissional, constituindo-se como meio incipiente para o surgimento de problemas de saúde mental por parte dos professores, como a SB. Assim, medidas de prevenção que estimulem o trabalho e o engajamento também podem minimizar as dificuldades e favorecer o desempenho das atividades docentes.

\section{REFERÊNCIAS}

ARANTES, V. A. Afetividade, cognição e moralidade na perspectiva dos modelos organizadores do pensamento. In: ARANTES, V. A afetividade na escola: alternativas teóricas e práticas. São Paulo: Summus. 2003.

ARONSSON, G. et al. A systematic review including meta-analysis of work environment and burnout symptoms. BMC Publ. Health, v. 17, n. 1, p. 264, 2017.

BATISTA, J. B. V. et al. Prevalência da Síndrome de Burnout e fatores sociodemográficos e laborais em professores de escolas municipais da cidade de João Pessoa, PB. Rev. Bras. Epidemiol., v. 13, p. 502-512, 2010.

BRASIL. Associação Brasileira de Empresas de Pesquisa (ABEP). Critério de classificação econômica. 2016. Disponível em: http://www.abep.org/criterio-brasil. Acesso em: 11 nov. 2020.

CAMPOS, J. A. D. B.; MAROCO, J. Maslach Burnout inventory-student survey: Portugal-Brazil crosscultural adaptation. Rev. saude Publ., v. 46, p. 816-824, 2012.

CARLOTTO, M.S. A síndrome de burnout e o trabalho docente. Psicol. Est., v. 7, n. 1, p. 21-29, 2002. 
CARLOTTO, M. S. Síndrome de Burnout em professores: prevalência e fatores associados. Psicol. teoria Pesq., v. 27, n. 4, p. 403-410, 2011.

CARLOTTO, M. S.; CÂMARA, S. G. Análise fatorial do Maslach Burnout Inventory (MBI) em uma amostra de professores de instituições particulares. Psicol. Est., v. 9, n. 3, p. 499-505, 2004.

CARLOTTO, M. S.; PALAZZO, L. S. Factors associated with burnout's syndrome: an epidemiological study of teachers. Cad. saude publ., v. 22, n. 5, p. 1017-1026, 2006.

CHOPRA, P. Mental health and the workplace: issues for developing countries. Int. J. Ment. Health Sy., v. 3, n. 1, p. 4, 2009.

DREHER, M. et al. Physical activity and its effects on burnout syndrome: A systematic review.

Laryngorhinootologie, v. 99, n. 2, p. 85-95, 2020.

DUKE, N. N. et al. Institutional factors associated with burnout among assistant professors. Teach. Learn. Med., v. 32, n. 1, p. 61-70, 2020.

ESTEVES-FERREIRA, A. A. et al. Avaliação comparativa dos sintomas da síndrome de burnout em professores de escolas públicas e privadas. Rev. Bras. Educ., v. 19, p. 987-1002, 2014.

GATTI, B. A. Os professores e suas identidades: o desvelamento da heterogeneidade. Cad. Pesq., n. 98, p. 85-90, 1996.

GOMES, A. et al. Occupational stress in teaching: a study with high school teachers. Psicol. Soc., v. 22, n. 3, p. 587-597, 2010.

GURSEL, M. et al. An analysis of burnout and job satisfaction between Turkish headteachers and teachers. Eur. J. Psychol. Educ., v. 17, n. 1, p. 35-45, 2002.

HACKMAN, J. R.; OLDHAM, G. R. Development of the job diagnostic survey. J. App. Psychol., v. 60, n. 2, p. 159, 1975.

LEITE, T. I. A. et al. Prevalence and factors associated with burnout among university professors.

Rev. Bras. Med. Trab., v. 17, n. 2, p. 170-179, 2019.

LEVY, G. C. T. M. et al. A. Síndrome de Burnout em professores da rede pública. Production, v. 19, n. 3, p. 458-465, 2009. 
LEWIS, R. Teachers Coping with the Stress of Classroom Discipline. Soc. Psychol. Educ., v. 3, n. 3, p. 155-171, 1999.

LUZ, J. G. et al. Implications of the environment, conditions and organization of work on teacher health: a systematic review. Cien. saude Col., v. 24, n. 12, p. 4621-4632, 2019.

MANDOLESI, L. et al. Effects of physical exercise on cognitive functioning and wellbeing: biological and psychological benefits. Front. Psychol., v. 9, p. 509, 2018.

MASLACH, C.; JACKSON, S. E.; LEITER, M. P.; SCHAUFELI, W. B. et al. Maslach burnout inventory. Consulting psychologists, 1986.

MASLACH, C.; JACKSON, S. The Measurement of Experienced Burnout. J. Org. Behav., v. 2, p. 99113, 1981.

MENDES, M. L. M. A precarização do trabalho docente e seus efeitos na saúde dos professores da rede municipal de ensino do Recife. Rev. Humanae, v. 9, n. 1, p. 1-18, 2015.

MESSIAS, I. M. O. et al. Burnout syndrome in teachers of a public school in Petrolina-PE. Braz. J. Develop., v. 5, n. 5, p. 3856-3866, 2019.

OMS - Organização Mundial da Saúde. The world health report 2000: health systems: improving performance. Genebra: OMS. 2000. Disponível em: https://www.who.int/whr/2000/en/. Acesso: 11 nov. 2020.

OUELLETTE, R.R. et al. Teacher job stress and satisfaction in urban schools: Disentangling individual-, classroom-, and organizational-level influences. Behav. Ther., v. 49, n. 4, p. 494-508, 2018.

RAUSCH, R. B.; DUBIELLA, E. Fatores que promoveram mal ou bem-estar ao longo da profissão docente na opinião de professores em fase final de carreira. Rev. Diál. Educ., v.13, n. 40, p. 10411061, 2013.

ROBBINS, S. et al. Comportamento organizacional: teoria e prática no contexto brasileiro. São Paulo: Pearson Prentice Hall, 2010.

SMETACKOVA, I. et al. Teachers between job satisfaction and burnout syndrome: what makes difference in Czech elementary schools. Front. Psychol., v. 10, p. 2287-2287, 2019.

TETRICK, L. E. et al. Prevention perspectives in occupational health psychology. In: ANTONIOU, A. S. G.; COOPER, C. L. (ed.) New horizons in management. Research companion to organizational health psychology. Cheltenham: Edward Elgar Publishing. 2005. 
1 Graduanda em Psicologia, Faculdade de Psicologia, Universidade Federal de Pelotas.

E-mail: ingridcandia2003@yahoo.com.br

2 Psicólogo, Mestre em Saúde e Ambiente, Programa de Pós-Graduação em Saúde e Comportamento, Universidade Católica de Pelotas. E-mail: igosv@hotmail.com

3 Graduando em Odontologia, Faculdade de Odontologia, Universidade Federal de Pelotas.

E-mail: mathsantos.f@gmail.com

4 Psicóloga, Doutora em Saúde e Comportamento, Programa de Pós-Graduação em Saúde e Comportamento, Universidade Católica de Pelotas.

E-mail: ana.laura@ufpel.edu.br 